\title{
Schistosoma mansoni Infections in Young Children: When Are Schistosome Antigens in Urine, Eggs in Stool and Antibodies to Eggs First Detectable?
}

\author{
J. Russell Stothard ${ }^{1 *}$, Jose C. Sousa-Figuereido ${ }^{1,2}$, Martha Betson ${ }^{1}$, Moses Adriko ${ }^{3}$, Moses Arinaitwe ${ }^{3}$, \\ Candia Rowell ${ }^{3}$, Fred Besiyge ${ }^{3}$, Narcis B. Kabatereine ${ }^{3}$
}

1 Wolfson Wellcome Biomedical Laboratories, Department of Zoology, Natural History Museum, London, United Kingdom, 2 Department of Infectious and Tropical Diseases, London School of Hygiene and Tropical Medicine, London, United Kingdom, $\mathbf{3}$ Vector Control Division, Ministry of Health, Kampala, Uganda

\begin{abstract}
Background: In Uganda, control of intestinal schistosomiasis with preventive chemotherapy is typically focused towards treatment of school-aged children; the needs of younger children are presently being investigated as in lakeshore communities very young children can be infected. In the context of future epidemiological monitoring, we sought to compare the detection thresholds of available diagnostic tools for Schistosoma mansoni and estimate a likely age of first infection for these children.

Methods and Findings: A total of 242 infants and preschool children (134 boys and 108 girls, mean age 2.9 years, minimum 5 months and maximum 5 years) were examined from Bugoigo, a well-known disease endemic village on Lake Albert. Schistosome antigens in urine, eggs in stool and host antibodies to eggs were inspected to reveal a general prevalence of 47.5\% ( $\left.\mathrm{Cl}_{95} 41.1-54.0 \%\right)$, as ascertained by a positive criterion from at least one diagnostic method. Although children as young as 6 months old could be found infected, the average age of infected children was between $3 \frac{1 / 4}{4}-3 / 4$ years, when diagnostic techniques became broadly congruent.

Conclusion: Whilst different assays have particular (dis)advantages, direct detection of eggs in stool was least sensitive having a temporal lag behind antigen and antibody methods. Setting precisely a general age of first infection is problematic but if present Ugandan policies continue, a large proportion of infected children could wait up to 3-4 years before receiving first medication. To better tailor treatment needs for this younger ageclass, we suggest that the circulating cathodic antigen urine dipstick method to be used as an epidemiological indicator.
\end{abstract}

Citation: Stothard JR, Sousa-Figuereido JC, Betson M, Adriko M, Arinaitwe M, et al. (2011) Schistosoma mansoni Infections in Young Children: When Are Schistosome Antigens in Urine, Eggs in Stool and Antibodies to Eggs First Detectable? PLoS Negl Trop Dis 5(1): e938. doi:10.1371/journal.pntd.0000938

Editor: Kirsten E. Lyke, University of Maryland School of Medicine, United States of America

Received May 25, 2010; Accepted December 4, 2010; Published January 4, 2011

Copyright: (c) 2011 Stothard et al. This is an open-access article distributed under the terms of the Creative Commons Attribution License, which permits unrestricted use, distribution, and reproduction in any medium, provided the original author and source are credited.

Funding: The work has been supported by the Wellcome Trust as a project grant awarded to JRS and NBK. The funders had no role in study design, data collection and analysis, decision to publish, or preparation of the manuscript.

Competing Interests: The authors have declared that no competing interests exist.

*E-mail: r.stothard@nhm.ac.uk

\section{Introduction}

Throughout the last decade several large-scale preventive chemotherapy campaigns, waged against neglected tropical diseases, have progressively scaled up operations to reach nationwide coverage levels in Uganda [1,2]. For control of intestinal schistosomiasis, as caused by Schistosoma mansoni infection, an active monitoring and surveillance programme, set within the national control programme (NCP), has provided important disease-specific information, assessing the impact of treatment upon the recipient population, as well as, re-alignment of original control objectives first set forth in 2003 [3,4].

Following WHO guidelines, mass-drug administration of praziquantel (PZQ) is typically focused towards treatment of school-aged children ( $\geq 6$ years) and adults who reside within disease endemic regions $[5,6]$. PZQ is provided free of charge by the NCP and analysis of school and(or) community treatment registers has shown that several million people have received at least one annual treatment of PZQ within the last five years $[1,7]$. Although this represents a considerable achievement, targeted epidemiological surveys have revealed that coverage is incomplete as in certain areas, e.g. shoreline environments of Lakes Victoria and Albert, large numbers of preschool-aged children ( $\leq 5$ years) and infants ( $\leq 1$ years) are infected with $S$. mansoni and have been largely overlooked by the treatment campaign $[8,9,10]$.

To ensure that this unfortunate health inequality does not persist the treatment needs of younger children are being assessed and we have recently called for formal inclusion of these young children within the Ugandan NCP [11]. It can be safely assumed, for example, that mass-treatment initiatives are vital in most in shoreline villages where infections can be common. Given the geographical focality of schistosomiasis and itinerancy of lakeshore communities, however, an important future challenge for the NCP is collection of sufficient disease-specific information to better tailor local drug needs and set parameters for subsequent programme monitoring [12,13]. Attention will therefore focus upon those 


\section{Author Summary}

In sub-Saharan Africa, intestinal schistosomiasis is a debilitating disease caused by a worm infection. To arrest disease progression, de-worming medications are given out, often en masse, to school-aged children. In Uganda, however, much younger children can be infected, and in lakeshore communities both infants and pre-school children can already show signs and symptoms of intestinal schistosomiasis. To change de-worming practices, further information on the occurrence of infections in these younger is needed for evidence-based decision making. Our study applied current methods of disease diagnosis to better define the 'age of first infection' and estimate general infection prevalence within a diseaseendemic village. Up to $50 \%$ of young children were clearly shown to have schistosomiasis and could likely wait up to 3-4 years before obtaining first treatment if present deworming policies are not changed. In the context of identifying future treatment needs, we propose that antigen detection methods are most suitable.

sections of villages where young children are frequently bathed in freshly drawn lake water or are within range of regular ambulation to the lake margins.

Owing to the unique natural history and developmental biology of schistosomes within the mammalian host [14], accurate identification of infected cases is challenging [15], even more so in the younger child where the founding worm population has only recently established and begun to mature. Before female worms develop their full egg-laying capacity, sporadic deposition of eggs may take place with a proportion of these being voided into the bowel lumen and ejected in faeces whilst the remainder become trapped within the host's tissues [16]. Interacting with this are also the beginnings of the child's innate and adaptive immune responses to excretory-secretory products of the worms themselves, as well as these responses being primed or modulated by maternally induced effects, for example, during pregnancy and(or) breastfeeding $[17,18,19,20]$. It is also of particular note that the child's immune system is in a maturing flux of recognition between self- and nonself epitopes [21] and the efficacy of PZQ, which is poor against immature worms of S. mansoni [22], is only starting to be explored in this ageclass [11]. From a general diagnostic perspective as existing tools are sub-optimal, improvement of methods and techniques for detection of intestinal schistosomiasis continues [15] but in the context of the younger child, it is not yet clear which of the present methods, or combinations thereof, is either most appropriate or applicable for routine use within the NCP.

We therefore report on a field-based study which attempted to determine the age of first infection in very young children with available techniques and also estimate, as accurately as possible, the general prevalence of intestinal schistosomiasis within this ageclass from a typical lakeshore community. The performance of methods that detect schistosome - antigens in urine, antibodies to egg antigens in serum and eggs in stool - was compared. For ease of comparison, our methods are subsequently referred to as: an antigen detection method (ADM), an indirect egg detection method (IEDM) and a direct egg detection method (DEDM), respectively.

\section{Materials and Methods}

This field study was carried out in April 2009 in Bugoigo on Lake Albert (GPS co-ordinates, N $01^{\circ} 54^{\prime} .481^{\prime \prime}$, E $31^{\circ} 24^{\prime} .597^{\prime \prime}$ ), a fishing village impoverished both in terms of sanitation and hygiene that has been the location of several previous research/ control studies on intestinal schistosomiasis [23,24,25,26]. Prevalence of infection within local school-aged children has been continuously high $(>50 \%)$ despite annual chemotherapy [27] and infections in infants and preschool children were first formally recorded in July 2007 [11].

\section{Study location and participants}

Owing to itinerancy, the exact number of inhabitants in Bugoigo is not precisely known but is likely in the region of several thousand. The village contains up to three thousand traditional hut dwellings which stretch 3-4 km along the lakeshore and up to $1-2 \mathrm{~km}$ inland. Sanitation and hygiene in this village is minimal with few potable water sources and insufficient pit latrines. Household water is typically drawn directly from the lake at specific collection points and then taken back to each homestead in plastic jerry cans for subsequent domestic use. These lakeshore margins, like elsewhere on Lake Albert, provide conducive aquatic habitats for Biomphalaria spp., the intermediate snail hosts of $S$. mansoni, and can be found throughout the year, although infected snails vary in numbers seasonally $[28,29]$.

The immediate and longer-term objectives of this study were explained to the local community mobiliser who identified a total of 134 mothers that were willing to participate, bringing up to two of their infants/preschool children ( $\leq 5$ years of age), and attend the two-day clinic commencing on the following day. After obtaining written informed consent from each mother on her own behalf and on behalf of her child(ren), urine, stool and fingerprick blood samples were obtained from all participants on the first day of the clinic. Mothers were then asked a suite of detailed questions recording their demography and water contact behaviours (the questionnaire is available upon request to the corresponding author).

After receipt of the second-day stool (and urine sample), all participants, regardless of their infection status, were treated for schistosomiasis and soil-transmitted helminthiasis with PZQ (40 mg/kg) (CIPLA, Mumbai, UK) and $400 \mathrm{mg}$ albendazole (GSK, Uxbridge, UK) under medical supervision in conditions typical of mass-drug administration [30]. For smaller children, a chewable albendazole half-tablet $(200 \mathrm{mg}$ ) was given and PZQ tablets were first crushed in orange juice before being administrated by spoon-feeding by their mother under supervision. The diagnostic findings for schistosomiasis here are reported for the children only.

\section{Schistosome antigens in urine (ADM)}

Each child's urine sample was visually inspected for macrohaematuria/turbidity and a random sample was tested for microhaematuria with Hemastix (Bayer, UK) to exclude the possibility of urinary schistosomiasis or other active urinary tract infections. A $50 \mu \mathrm{l}$ aliquot was then tested for the presence of schistosome circulating cathodic antigen (CCA) using a commercially available lateral flow immuno-chromatographic urine dipstick (Rapid Medical Diagnostics, Pretoria, RSA) originally developed in Holland [31]. On a subset of 90 children, urine-CCA tests were performed in duplicate to assess variation between dipsticks.

To facilitate better recording of the visual intensity of the CGA reaction band within the test zone, results were visually graded against a reference chart for: trace, single $(+)$, double $(++)$ and triple $(+++)$ positive reactions [32]. When creating binomial variables to depict infection status according to CCA, two variations were taken into account: the first considering trace results as negative infection status and the second considering trace 
results as positive infection status. The urine CGA reagent strip is referred to as an ADM (antigen detection method) from now on.

\section{Antibodies to soluble egg antigens (IEDM)}

A commercially available ELISA kit (IVD Inc.; Carlsbad, USA) was used to test for host antibodies (IgG/M) to soluble egg antigens (SEA) according to manufacturer's instructions. Approximately $75 \mu$ of finger-prick blood was taken from each child and serum was harvested, then diluted 1:40 with specimen dilution buffer before loading a total of $100 \mu$ into each ELISA microwell [11]. Positive and negative control sera were included on each batch of testing. Upon completion, each ELISA plate was placed on a white card and the colour within each microwell (ranging from colourless to yellow) was recorded by visual inspection. Positive reactions were classified either as trace (faint yellow), single $(+$, light yellow), double $(++$, yellow) or triple $(+++$, dark yellow) upon visual comparison with the control sera. The SEAELISA is referred to as an IEDM (indirect egg detection method) from now on.

\section{Direct egg-detection methods in stool (DEDM)}

Three parasitological methods Kato-Katz, percoll and FLOTAC, henceforth referred to as direct egg detection methods (DEDMs), were attempted on each stool specimen to visualise eggs. However, owing to the differing amounts of stool required for each technique, it was not always possible to assemble a complete data set for every child with each of these three methods.

Duplicate Kato-Katz (K-K) thick smears $(41.7 \mathrm{mg})$ were made from first and second day stool samples $(\mathcal{N}=242$ children) [33]. The four faecal smears were each examined under the microscope at x100, schistosome eggs were counted and later expressed as eggs per gram (epg) of faeces. Infection intensity was classified as light (1-100 epg), medium (101-400 epg) and heavy (>400 epg) infections according to WHO guidelines [5]. The methodology of Eberl [34] using sedimentation of schistosome eggs by centrifugation through a solution of percoll (Percoll $77237(1.130 \mathrm{~g} / \mathrm{ml})$, Fluka, Sigma-Aldrich Chemie $\mathrm{GmbH}$, Switzerland) was also implemented on-site to visualize eggs $(\mathcal{N}=96$ children on first day stool). The egg-floatation procedure known as FLOTAC [35] was performed off-site back in Kampala on a formalin-fixed stool specimen archive $(\mathcal{N}=191$ children taken from the second day stool) whereby schistosome eggs are collected by floatation centrifugation through a solution of zinc sulphate at specific gravity of 1.35 .

\section{Data handling and statistical analyses}

Data were collected from each individual using pro-forma data sheets, which were then transferred into electronic format using Microsoft Excel. The data thus collated were analysed using MS Excel and R statistical package version 2.8.0 [36]. For prevalence data and diagnostic parameters, 95\% confidence intervals $\left(\mathrm{CI}_{95}\right)$ were estimated using the exact method [37]. Prevalence comparisons were performed using (one-tailed) Fisher's exact modification of the $2 \times 2$ chi-squared test [38]. For infection intensity values, the arithmetic mean of positive cases was chosen as the measure of central tendency. Data from the FLOTAC and percoll methods were analysed by combining with $\mathrm{K}-\mathrm{K}$ results and revising the diagnostic criterion so individuals were considered positive if an egg was detected by at least one DEDM.

The diagnostic performances of the ADM (including and excluding trace reactions as a positive diagnosis) and IEDM were tested qualitatively as a rapid diagnostic for intestinal schistosomiasis, considering DEDMs as the 'gold-standard'. Additionally, a third 'gold standard' was created using data from the ADM (including and excluding trace reactions as positive diagnoses) against which to test IEDM data $(\mathcal{N}=242)$. Diagnostic sensitivity, specificity, positive predictive value (PPV) and negative predictive value (NPV) were calculated according to the different 'gold standards' [38]. The diagnostic powers of ADM and IEDM were calculated using all individuals, and then segregated by sex or age ( $\leq 3$ years of age versus $>3$ years of age). $P$-values $<0.05$ were considered indicative of statistical significance [38].

\section{Ethical approvals}

Approvals for this study were granted by the Ugandan Council for Science and Technology and the London School of Hygiene and Tropical Medicine (application numbers 06.45 and 5538.09). After sensitisation of the local community to the study objectives, verbal assent was first requested from each mother which was then formalised upon written informed consent (for her and behalf of her child), as either a thumbprint or signature on data recording sheet. This was witnessed by a Vector Control Division Officer. PZQ treatment $(40 \mathrm{mg} / \mathrm{kg}$ ) was offered to all study participants irrespective of their infection status.

\section{Results}

A complete data set for the ADM, IEDM and Kato-Katz examinations was obtained from a total of 242 children (134 boys: 108 girls, mean age 2.9 years, minimum 5 months and maximum 5 years). However, owing to insufficient amounts of stool available, the FLOTAC and percoll methods could only be performed on 191 and 96 children, with the former and latter finding 4 and 2 additional egg-positive cases, respectively.

\section{Estimating infection prevalence}

The prevalence of intestinal schistosomiasis estimated by each diagnostic method, and combinations thereof, is shown in Table 1 and Fig. 1. Prevalence inferred by DEDM, ADM (including trace reactions as positive diagnoses) and IEDM (considering traces as negatives) were: $24.4 \%, 42.6 \%$ and $45.9 \%$. Of the children who were egg-positive by K-K, three quarters had 'light' intensity infections. Girls were equally as likely as boys to be diagnosed positively for intestinal schistosomiasis by ADM (Odds Ratio (OR) $=1.06, \mathrm{p}=0.90)$ and DEDM examinations $(\mathrm{OR}=0.72, \mathrm{p}=0.29)$. Children under the age of three, however, were less likely to be positive by $\operatorname{ADM}(\mathrm{OR}=0.51, \mathrm{p}=0.016)$ or by $\mathrm{DEDM}(\mathrm{OR}=$ $0.26, \mathrm{p}<0.0001)$ than their older counterparts. The prevalence of positives by IEDM was $45.9 \%$. General prevalence inferred by pooling DEDM and IEDM was $47.7 \%$, with no further change in prevalence when ADM was then added, see Fig. 1. There was no discordance between duplicate CCA testing for negative or positive classifications (data not shown).

\section{Ages of becoming first positive}

The age of first positive (AFP) for each method is presented in Table 1. For DEDM, the youngest child with eggs in stool was 9 months old, with medium and heavy infections found at 3 and 5 years of age, respectively. For ADM, trace reactions, single, double and triple positives were found in an ascending series of 6 months, 9 months, 11 months and 2 years of age, respectively. For IEDM, trace reactions began at 5 months of age while single, double and triple positive reactions were found in children as young as 6 months, 1 year and 9 months old, respectively. All tests concur on a mean age of first infection within the third year of life. ADM detected infections slightly ahead of I/DEDMs (3.2 years v. 3.4 years v. 3.7 years, respectively). The order of this temporal series is 
Table 1. Prevalence and intensity of intestinal schistosomiasis and age of first positive by each diagnostic method.

\begin{tabular}{|c|c|c|c|c|c|}
\hline & No. tested & Prevalence (\%) & $\mathrm{Cl}_{95}(\%)$ & Mean epg (of positives) & AFP $^{\$}$ \\
\hline DEDM (Two-sample double-smear Kato-Katz) & 242 & 21.9 & $16.9-27.6$ & 86.4 & 9 months \\
\hline KK light intensity (<100 epg) & & 16.9 & $12.4-22.3$ & & 9 months \\
\hline KK medium intensity (100-399 epg) & & 4.5 & $2.3-8.0$ & & 3 years \\
\hline KK heavy intensity (>399 epg) & & 0.4 & $0.0-2.3$ & & 5 years \\
\hline ADM (CCA incl. trace) & 242 & 42.6 & $36.3-49.1$ & NA & 6 months \\
\hline + reaction & & 17.8 & $13.2-23.2$ & & 9 months \\
\hline ++ reaction & & 11.6 & $7.8-16.3$ & & 11 months \\
\hline +++ reaction & & 1.7 & $0.5-4.2$ & & 2 years \\
\hline IEDM (SEA-ELISA) & 242 & 45.9 & $39.5-52.4$ & NA & 6 months \\
\hline + reaction & & 15.3 & $11.0-20.5$ & & 6 months \\
\hline ++ reaction & & 19.4 & $14.6-25.0$ & & 1 year \\
\hline+++ reaction & & 11.2 & $7.5-15.8$ & & 9 months \\
\hline DEDMs (all*) & 242 & 24.4 & $19.1-30.3$ & NA & NA \\
\hline$I E D M+D E D M+A D M$ & 242 & 47.5 & $41.1-54.0$ & NA & NA \\
\hline
\end{tabular}

${ }^{\$}$ Age of first positive.

*Note not all 242 children were examined with percoll and FLOTAC (see methodology).

doi:10.1371/journal.pntd.0000938.t001

largely concordant with an absolute minimum age of becoming first positive.

\section{Cross-tabulations of diagnostic scores}

In the absence of a genuine 'gold standard' where the infection status of each child is precisely known, it is necessary to explore relationships between diagnostic scores and infection intensities empirically, and to cross-tabulate diagnostic permutations by investigation. There was negligible variation in diagnostic performance of all protocols tested when classifying the data according to sex and age (data not shown) and general trends were reported from now on.

Plotting the relationship between ADM and DEDM revealed some immediate trends, Fig. 2. Whilst there were children positive for $\mathrm{ADM}$ who were egg-negative, as the epg increases there was a corresponding increase in the proportion of positive ADM tests and once medium/heavy intensity infections were reached, all

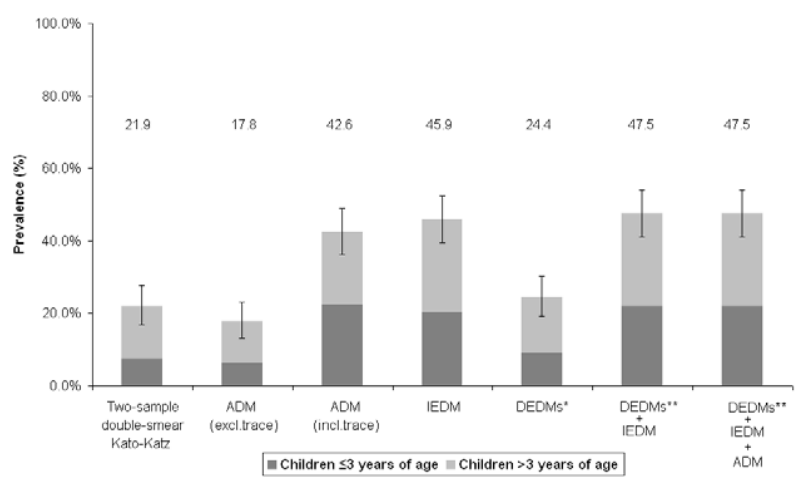

Figure 1. Barchart of the prevalence of intestinal schistosomiasis by each diagnostic technique (as well as pooling techniques, DEDM* representing results obtained from KatoKatz, Percoll and FLOTAC methods) for the examined children. Note that the DEDMs under estimate infection prevalence and the addition of ADM to DEDMs/IEDM data did not further increase cumulative infection prevalence.

doi:10.1371/journal.pntd.0000938.g001
ADM tests were clearly positives, see Fig. 2A. Plotting the faecal epg of each child against the intensity of the corresponding ADM test further revealed this positive association, see Fig. 2B.

Considering the relationship between IEDM and DEDM revealed similar trends, see Fig. 3. Despite some children being positive for IEDM while being egg-negative, as the faecal epg increases there was a corresponding increase in the IEDM reaction strength, with all medium/heavy intensity infections diagnosed as clear strong positives (Fig. 3A \& B).

The relationship between ADM and IDEM was less clear-cut. Children who were ADM negative or trace had a median negative (or trace) IEDM reaction, but the proportionate increase of ADM positives with rising IEDM designations of positive $(+)$ or strong positives $(++/+++)$ was not as great as that seen with DEDM. For example, nearly $40 \%$ of children who were IEDM strong positive elicited a negative ADM reaction, Fig. 4A. As the intensity of the $\mathrm{ADM}$ result stepped up towards double and triple positive reactions, this typically corresponded better with increasing IEDM classifications, Fig. 4B.

Using available data it was possible to conduct an exploration of diagnostic performances of the ADM and IEDM versus DEDM and against each other (Table 2). First, considering an ADM trace reaction to be an infection negative and comparing with positive diagnosis by at least one of the DEDMs, the ADM had a sensitivity of $59.3 \%$, specificity of $95.6 \%$, PPV of $81.4 \%$ and NPV of $87.9 \%$. When considering an $\mathrm{ADM}$ trace reaction to be a positive infection, and comparing to diagnosis by at least one of the DEDMs, ADM had a sensitivity of $81.4 \%$, specificity of $69.9 \%$, PPV of $46.6 \%$ and NPV of $92.1 \%$.

The IEDM when compared to diagnosis by DEDM, demonstrated a sensitivity of $93.2 \%$, specificity of $69.4 \%$, PPV of $49.5 \%$ and NPV of $96.9 \%$. For details on the performance of the ADM or IEDM compared to diagnosis by all DEDMs (using a subset of the data), and for $\mathrm{CI}_{95}$ around each value, see Table 2 .

\section{Discussion}

With limited access to safe water sources, and high levels of local transmission of S. mansoni, conditions in Bugoigo are particularly 


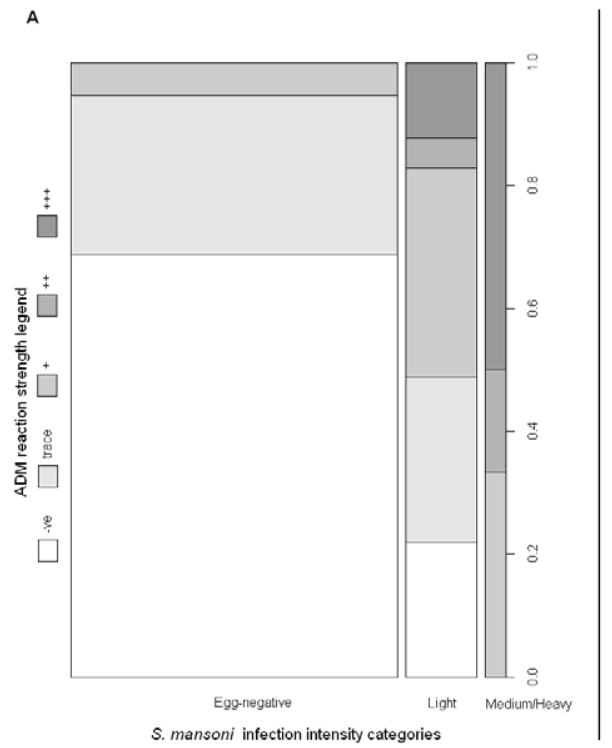

B

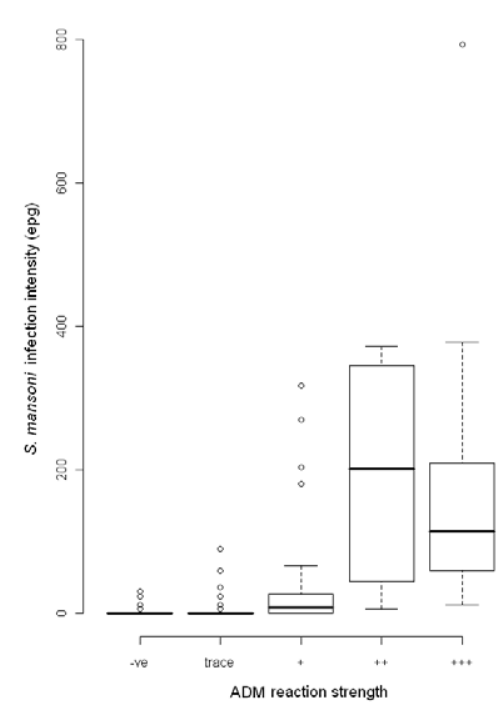

Figure 2. Comparisons of ADM and DEDM. Figure 2A Rectangular bar chart representing egg infection intensity classifications (as calculated by Kato-Katz) versus reaction intensity of the ADM (visual strength of the CCA urine dipstick test band). Figure 2B Boxplot of the egg faecal epg against $A D M$ reaction intensity shows a positive increasing association. doi:10.1371/journal.pntd.0000938.g002

conducive for young children to acquire S. mansoni infections, and from a very early age. Approximately half of our children had intestinal schistosomiasis. As might be expected, regardless of techniques used, there was an obvious positive association between increasing diagnostic patency of infection with increasing age of the child. Presumably this was resultant from a progressive temporal accumulation of antigens, eggs and antibodies. Congruence between diagnostic methods became most apparent in children between $3 \frac{1 / 4}{-3} \frac{3 / 4}{4}$ years of age, broadly consistent with the overall mean age of infected children within our sample. Prevalence of intestinal schistosomiasis in children under 3 years of age, however, was 35.5\% $\left(\mathrm{CI}_{95} 27.9-43.8 \%\right)$ and other studies have also revealed that schistosomiasis in very young children can be common $[9,11,39]$.

While egg excretions of these children were of 'light' intensity, such infected children will not normally receive praziquantel treatment until they have either entered primary school or if the NCP now formally extends its treatment remit to include this ageclass. Thus an infected child could therefore wait up to 3-4 years before receiving first treatment, and with this may have already entered a more 'chronic' stage of disease [40,41,42,43]. For example, earlier clinical and ultrasound studies in Uganda in
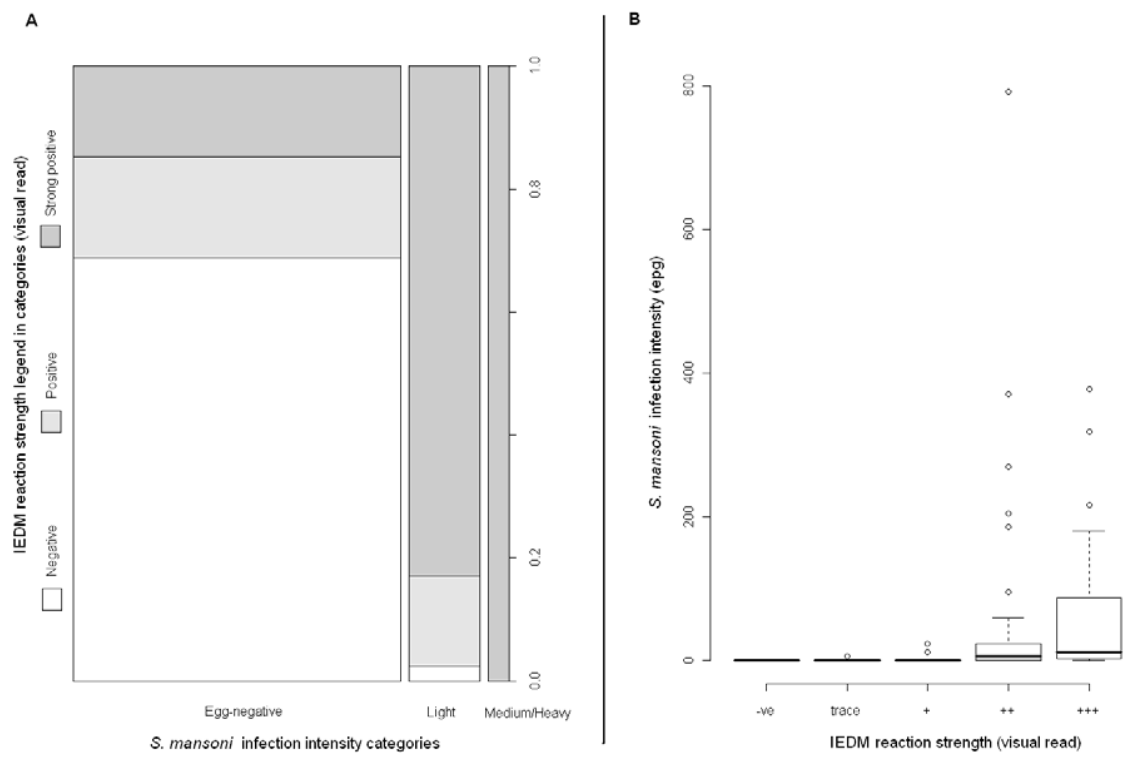

Figure 3. Comparisons of IEDM and DEDM. Figure 3A Rectangular bar chart representing egg infection intensity classifications (as calculated by Kato-Katz) versus reaction intensity of the IEDM (visual strength of the SEA-ELISA test well). Figure 3B Boxplot of the egg faecal epg against IEDM reaction intensity shows a positive increasing association.

doi:10.1371/journal.pntd.0000938.g003 


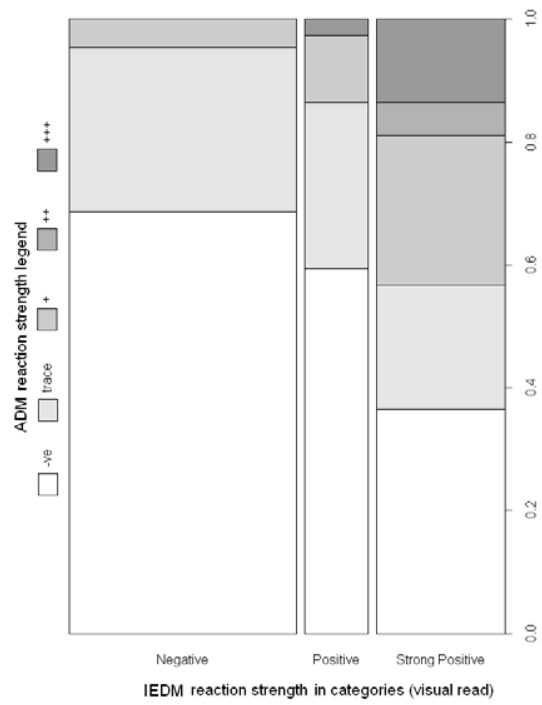

B

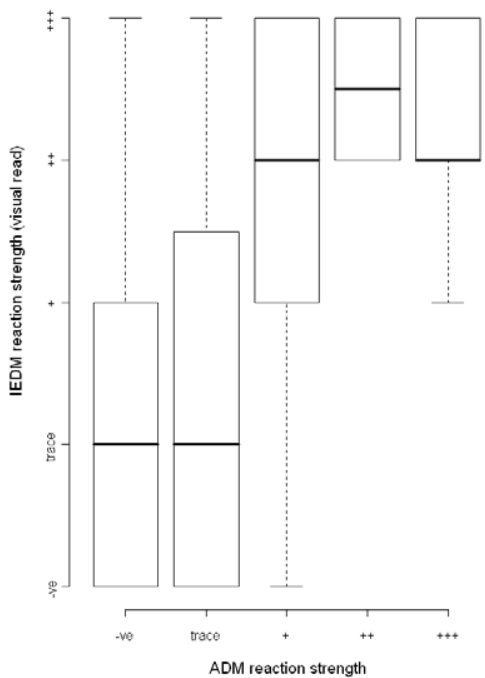

Figure 4. Comparisons of ADM and IEDM. Figure 4A Rectangular bar chart representing ADM intensity classifications (visual strength of the CCA urine dipstick test band) versus reaction intensity of the IEDM (visual strength of the SEA-ELISA test well). Figure 4B Boxplot of the IEDM reaction intensity against ADM shows a positive increasing association but the relationship is less clear-cut than that shown in Figures $\mathbf{2 B} \& \mathbf{3 B}$. doi:10.1371/journal.pntd.0000938.g004

children aged 6 and above, have shown significant hepatosplenomegaly (i.e. putative morbidity from intestinal schistosomiasis), and while they have not yet developed pipe-stem liver fibrosis, up to $15 \%$ can have diffusely echogenic livers with pocketed foci, typical of image pattern B ('the starry sky' classification) [3,27]. Without medication, it is likely that these preschool children will progress towards 'moderate' infection intensities before they become of school age. This might better explain the observations of Balen et al. that many adolescent Ugandan children have surprisingly severe intestinal schistosomiasis [44]. Although it is not yet proven that infection in very early childhood leads to heightened morbidity in later childhood and adolescence, this scenario appears plausible.

From animal models, it is known that only a fraction of penetrating cercariae successfully migrate to, and later mature in, the hepatic portal system. After adult worms reach full fecundity, schistosome eggs can be found in stool around 6 weeks after cercarial exposure and it is commonly held that females of $S$. mansoni produce up to 100-300 eggs per day, although many fail to be voided into the faeces [16]. Given the insensitivity of DEDMs in stool [34], it is not surprising that false negatives are inferred and the low egg-detection threshold(s) likely contribute to the longer apparent lag of 7-8 months between infection and eggpatency apparent between experimental schistosomiasis and the situation in the field. Moreover, it should be noted that the relationship between excreted eggs in stool and worm burdens is not always straightforward [45] and that infected laboratory animals are typically exposed with a single substantive dosing of cercariae. By contrast, and in this natural setting, exposure and infection is likely a more gradual process, i.e. the so-called trickle infection dynamic [46], and our children are at least two orders of magnitude greater in body size than most animal models.

Table 2. Comparison of diagnostic scores by ADM and IEDM using DEDM (all) as 'gold standard'.

\begin{tabular}{|c|c|c|c|c|c|c|}
\hline \multirow[t]{2}{*}{ Diagnostic test } & \multirow[t]{2}{*}{ Diagnostic target } & \multirow[t]{2}{*}{$\mathbf{N}$} & \multirow{2}{*}{$\frac{\text { Sensitivity }}{\left(\%, \mathrm{Cl}_{95}\right)}$} & \multirow{2}{*}{$\frac{\text { Specificity }}{\left(\%, \mathrm{Cl}_{95}\right)}$} & \multirow{2}{*}{$\frac{P P V}{\left(\%, \mathrm{Cl}_{95}\right)}$} & \multirow{2}{*}{$\frac{N P V}{\left(\%, C l_{95}\right)}$} \\
\hline & & & & & & \\
\hline \multirow[t]{2}{*}{ ADM (excl. trace) } & $D E D M$ & 242 & 59.3 & 95.6 & 81.4 & 87.9 \\
\hline & & & $(45.8-71.9)$ & $(91.6-98.1)$ & $(66.6-91.6)$ & $(82.6-92.1)$ \\
\hline \multirow[t]{2}{*}{$A D M$ (incl. trace) } & $D E D M$ & 242 & 81.4 & 69.9 & 46.6 & 92.1 \\
\hline & & & $(69.1-96.3)$ & $(62.7-76.5$ & $(36.7-56.7)$ & $(86.3-96.0)$ \\
\hline \multirow[t]{6}{*}{ IEDM } & $D E D M$ & 242 & 93.2 & 69.4 & 49.5 & 96.9 \\
\hline & & & $(83.5-98.1)$ & $(62.2-76.0)$ & $(40.0-59.2)$ & $(92.4-99.2)$ \\
\hline & ADM (excl. trace) & 242 & 86 & 62.8 & 33.3 & 95.4 \\
\hline & & & $(72.1-94.7)$ & $(56.0-69.5)$ & $(24.7-42.9)$ & $(90.3-98.3)$ \\
\hline & ADM (incl. trace) & 242 & 60.2 & 64.7 & 55.9 & 68.7 \\
\hline & & & $(50.1-69.7)$ & $(56.2-72.7)$ & $(46.1-65.3)$ & $(60.0-76.5)$ \\
\hline
\end{tabular}




\section{An age of first infection?}

While some children were patently infected during the first year of life, others were not. Thus a sub-set of children exists with increased infection risk factors which we explain by the following synopsis. As children are born throughout the year, in a largely asynchronous fashion, whilst their initial age of first exposure to unsafe water might be broadly similar (i.e. within first few months of life as mothers begin to bathe them in jerry-can collected water or in the lake directly) their accumulated infection risk will not be equivalent owing the seasonality of local transmission factors and their particular timeframe of exposure within it contingent upon their mother's infant bathing and domestic water drawing practices [11].

Estimating this accumulated risk of infection reliably over the seasonal time frame of potential exposure is problematic as day-today variations within water collection times, its storage and actual domestic use (within each household) introduce many stochastic processes. Estimating cumulative infection risk is therefore easily confounded but an ad hoc investigation of infection risk associated with jerry-can collected water in June 2009, however, has confirmed that sentinel laboratory-bred mice could become infected to freshly drawn water [28]. Seasonal patterns, which operate in umbrella fashion over and above these specific-exposure patterns, no doubt effect this asynchronous age of first infection. Thus there will be no 'absolute age' of first infection but rather a 'range of ages' depending upon these intricate covariates of exposure. Only after a child has passed through sufficient 'windows of exposure', their probability of infection rises to an eventual certainty, after which, it is incumbent on the diagnostic tools to capture their parasitological status as accurately as possible.

\section{Comparison of diagnostic scores}

From first appearances the ADM looks to best capture and identify infections in early stage, especially when we consider trace results as putative infection positives. A contentious issue in the use of the CCA reagent strip has been the interpretation of the exact diagnosis of this 'trace' result which can be confounded by nonspecific inflammatory factors or breast-feeding [32,47]. Interpretation of 'trace' is more contentious when surveying children under three years of age, where worm burdens are presumably lower than what might be expected in their school-aged counterparts. Interestingly, the percentage change in prevalence estimated according to the CCA reagent strip when excluding and including trace results as a positive diagnosis is significantly larger in the very young children ( $\leq 3$ years of age) $-10.1 \%$ v. $36.2 \%$ $(+358 \%)$ - than in those aged four and five years of age $-30.1 \% \mathrm{v}$. $52.7 \%(+175 \%)$ which is fitting with our understanding of increasing worm burdens through time. Thus we postulate that using 'trace' as positive firmly points towards a future use of the urine CCA-dipstick as an early indicator of infections which are as yet to become egg- or antibody-patent. It is particularly notable that the prevalence based on the ADM, when considering trace as positive, is very close to that of IEDM (Fig. 1), yet the diagnostic performance with it was not particularly congruent (see Fig. 3 and Table 2) so we still have an incomplete understanding of this infection progression. The dynamics of other ADM have been explored elsewhere in the context of recently acquired infection but not in very young children [48].

The ADM showed very promising diagnostic performance and robust field performance with high sensitivity and NPV scores $(83.9 \%$ and $85.3 \%$, respectively) when we considered trace results as a positive diagnoses and very high specificity and PPV scores (95.5\% and $90.5 \%$, respectively) when we considered trace results as negative diagnoses. This bimodal use of the test criteria could be advantageous from a control perspective. For instance, if a confident estimate of the suspected occurrence of infections within a population is needed, one should consider trace results as positives. On the other hand, to monitor the prevalence of 'actual' infection, or rather more easily identify those who do not, one should consider trace results as negatives. The former would be important if treatments were to be given out en masse as triggered by exceeding an aggregated local prevalence threshold while the latter would be important if treatment were to be withheld in an individual patient setting on the basis of test and treat.

\section{Towards promotion of safe water}

With the gradual rise of infection prevalence in older children (over and above our asynchronous infection hypothesis), this trend must represent the spread of several risk factors, rather incipiently, across our cohort. Aggregation of infections in schistosomiasis is well-known [49] but it would be interesting to establish why approximately half of our study cohort had no evidence of infection despite living within the same village. As we were insufficiently aware of the exact locations of sampled households within the village, this could simply represent a cryptic spatial micro-patterning (i.e. these children who live slightly further away from the lake have less contact with viable cercariae) so we are now undertaking fine scale mapping of these individual households with GPS units. If, however, other causal factors could be identified and, perhaps more importantly, were these amenable to manipulation, it could lead to future infection mitigation measures.

Presently, within the NCP there are no health education materials targeted towards these mothers and their young children. More importantly and in terms of policy realignment of the NCP, a useful formal recommendation would be to initiate cross-sectorial activities with water and sanitation NGOs to improve immediately the domestic water quality at Bugoigo and elsewhere along the Lake Albert shoreline. Rather than focusing upon expensive infrastructure development, it could be achieved by introduction of simple water storage or modification measures. For example, as schistosome cercariae are an ephemeral larval stage, freshly drawn water can be rendered harmless for schistosomiasis by simple resting for $24 \mathrm{hrs}$, by crude filtration or by introduction of mild disinfectants [16]. Thus without initiating a better dialogue with these women of children bearing age through better public health education, mothers will remain sadly ignorant of the risks that making use of this unsafe water has for themselves and that of the future health of their child [11]. In this dialogue, the NCP should be receptive to explore which infection mitigation measures are best feasible and, by this token, help to provide safe water for domestic use which is well-received, implementable and effective.

\section{Epidemiological indicators and treatment needs}

It is evident that infants and preschool children in Bugoigo, and other similar lakeshore villages of Uganda [12], are living in need of treatment. However, addressing how these children could be best identified is not yet clear, as are epidemiological parameters which should be collected for estimating treatment needs and also impact assessment. For example, should mass-treatment of all infants/preschool children take place when a sub-sample of an equivalent age range has been proven to be infected, or should treatment be allocated based at an individual level using the result of a diagnostic test in a 'test and treat' setting?

It is outside the remit of this present paper to make a costeffectiveness calculation but a clear drawback of the IEDM method is that, whilst initially useful to establish if a child is 
infected (and there is no evidence in these data to suggest a passive maternal transfer of antibodies has been confounding), monitoring this parameter after treatment will be largely uninformative owing to residual antibody titres remaining after infection has putatively cleared. Thus IEDM is only useful at baseline but as an initial estimate of infection prevalence could be powerfully applied in identification and selection of villages, or sentinel locations, to first define the extent of the problem at intervention baseline. This of course assumes the majority of examined children can mount an antibody response and is not confounded by high levels of immune-suppression, by HIV for example, which is likely high in these fishing villages.

In contrast, both $\mathrm{ADM}$ and DEDMs have the potential ability to better track the dynamics of worm populations after treatment $[22,34]$ but the insensitivity of DEDMs is of particular concern. Put simply, numerous adult worms may reside within the host but are yet not depositing sufficient eggs to be visualised in stool on the day of sampling. Thus through lack of alternatives a pragmatic way forward would be to focus upon more widespread application of the ADM. The advantages of the ADM have been discussed elsewhere in the context of programmatic monitoring [14] but the future challenge will be for the NCP to meet the financial costs of using these rapid diagnostic tests in scale-up of operations. This is particularly true if these are to be used in a 'test and treat' setting when large numbers of tests would be utilized [14]. Given the low price of PZQ treatment, to maintain an affordable diagnosis versus treatment differential, a rational strategy would be to examine a sub-set of children and if local prevalence exceeded a given threshold, mass-treatment is advised. Such a strategy is presently

\section{References}

1. Fenwick A, Webster JP, Bosque-Oliva E, Blair L, Fleming FM, et al. (2009) The Schistosomiasis Control Initiative (SCI): rationale, development and implementation from 2002-2008. Parasitology 136: 1719-1730.

2. KolaczinskiJH, Kabatereine NB, Onapa AW, Ndyomugyenyi R, Kakembo AS, et al. (2007) Neglected tropical diseases in Uganda: the prospect and challenge of integrated control. Trends in Parasitology 23: 485-493.

3. Kabatereine NB, Brooker S, Koukounari A, Kazibwe F, Tukahebwa EM, et al. (2007) Impact of a national helminth control programme on infection and morbidity in Ugandan schoolchildren. Bulletin of the World Health Organization 85: 91-99.

4. Brooker S, Whawell S, Kabatereine NB, Fenwick A, Anderson RM (2004) Evaluating the epidemiological impact of national control programmes for helminths. Trends in Parasitology 20: 537-545.

5. WHO (2002) Prevention and Control of Schistosomiasis and Soil-Transmitted Helminthiasis. Report of a WHO Expert Committee. Geneva: World Health Organization.

6. Savioli L, Gabrielli AF, Montresor A, Chitsulo L, Engels D (2009) Schistosomiasis control in Africa: 8 years after World Health Assembly Resolution 54.19. Parasitology 136: 1677-1681.

7. Fleming FM, Fenwick A, Tukahebwa EM, Lubanga RGN, Namwangye H, et al. (2009) Process evaluation of schistosomiasis control in Uganda, 2003 to 2006 : perceptions, attitudes and constraints of a national programme. Parasitology 136: 1759-1769.

8. Johansen MV, Sacko M, Vennervald BJ, Kabatereine NB (2007) Leave children untreated and sustain inequity! Trends in Parasitology 23: 568-569.

9. Odogwu SE, Ramamurthy NK, Kabatereine NB, Kazibwe F, Tukahebwa E, et al. (2006) Schistosoma mansoni in infants (aged $<3$ years) along the Ugandan shoreline of Lake Victoria. Annals of Tropical Medicine and Parasitology 100: 315-326.

10. Stothard JR, Gabrielli AF (2007) Schistosomiasis in African infants and preschool children: to treat or not to treat? Trends in Parasitology 23: 83-86.

11. Sousa-Figueiredo JC, Pleasant J, Day M, Betson M, Rollinson D, et al. (2010) Treatment of intestinal schistosomiasis in Ugandan preschool children: Best diagnosis, treatment efficacy \& side-effects, and an extended praziquantel dosing pole. International Health 2: 103-113.

12. Brooker S, Kabatereine NB, GyapongJO, Stothard JR, Utzinger J (2009) Rapid mapping of schistosomiasis and other neglected tropical diseases in the context of integrated control programmes in Africa. Parasitology 136: 1707-1718.

13. Stothard JR (2009) Improving control of African schistosomiasis: towards effective use of rapid diagnostic tests within an appropriate disease surveillance model. Transactions of the Royal Society of Tropical Medicine and Hygiene 103: $325-332$ within the resources available to the NCP but best sample sizes and prevalence thresholds remain to be determined.

\section{Supporting Information}

Checklist S1 STROBE Checklist

Found at: doi:10.1371/journal.pntd.0000938.s001 (0.10 MB DOG)

\section{Acknowledgments}

We would like to thank several other members of the VCD field team for their help and assistance during the survey: Aaron, Andrina, Annet, Aida, David and Fiddi, as well as, local VCD assistants at Bugoigo camp: Chris, Perez, Caesar and Ashuman. We are grateful for the time and effort our mothers took to attend our clinic. JRS and MB are grateful to the staff of Giuseppe Cringoli and Laura Rinaldi's laboratory for their assistance and hospitality in Naples for training in use of FLOTAC. We thank David Rollinson for his helpful comments which improved this manuscript as well as those from the anonymous referees.

\section{Author Contributions}

Conceived and designed the experiments: J. Stothard, J. Sousa-Figuereido, N. Kabatereine. Performed the experiments: J. Stothard, J. SousaFiguereido, M. Betson, M. Adriko, M. Arinaitwe, C. Rowell, F. Besiyge. Analyzed the data: J. Stothard, J. Sousa-Figuereido, M. Betson. Contributed reagents/materials/analysis tools: J. Stothard, N. Kabatereine. Wrote the paper: J. Stothard, J. Sousa-Figuereido, M. Betson, M. Adriko, M. Arinaitwe, C. Rowell, F. Besiyge, N. Kabatereine.

14. Kusel JR, Al-Adhami BH, Doenhoff MJ (2007) The schistosome in the mammalian host: understanding the mechanisms of adaptation. Parasitology 134: $1477-1526$.

15. Bergquist R, Johansen MV, Utzinger J (2009) Diagnostic dilemmas in helminthology: what tools to use and when? Trends in Parasitology 25: 151-156.

16. Jordan P, Webbe G, Sturrock R (1993) Human schistosomiasis. Wallingford, England: CAB.

17. Friedman JF, Mital P, Kanzaria HK, Olds GR, Kurtis JD (2007) Schistosomiasis and pregnancy. Trends in Parasitology 23: 159-164.

18. Woolhouse MEJ, Mutapi F, Ndhlovu PD, Chandiwana SK, Hagan P (2000) Exposure, infection and immune responses to Schistosoma haematobium in young children. Parasitology 120: 37-44.

19. Butterworth AE (1990) Studies on human schistosomiasis: chemotherapy, immunity and morbidity. Annales de Parasitologie Humaine et Comparée 65: $53-57$.

20. Elliott AM, Kizza M, Quigley MA, Ndibazza J, Nampijja M, et al. (2007) The impact of helminths on the response to immunization and on the incidence of infection and disease in childhood in Uganda: design of a randomized, doubleblind, placebo-controlled, factorial trial of deworming interventions delivered in pregnancy and early childhood. Clinical Trials 4: 42-57.

21. Holt PG, Macaubas C (1997) Development of long term tolerance versus sensitisation to environmental allergens during the perinatal period. Current Opinion in Immunology 9: 782-787.

22. Utzinger J, N'Goran EK, N'Dri A, Lengeler C, Tanner M (2000) Efficacy of praziquantel against Schistosoma mansoni with particular consideration for intensity of infection. Tropical Medicine and International Health 5: 771-778.

23. Booth M, Vennervald BJ, Kabatereine NB, Kazibwe F, Ouma JH, et al. (2004) Hepatosplenic morbidity in two neighbouring communities in Uganda with high levels of Schistosoma mansoni infection but very different durations of residence. Transactions of the Royal Society of Tropical Medicine and Hygiene 98: 125-136.

24. de Moira AP, Fulford AJC, Kabatereine NB, Kazibwe F, Ouma JH, et al. (2007) Microgeographical and tribal variations in water contact and Schistosoma mansoni exposure within a Ugandan fishing community. Tropical Medicine \& International Health 12: 724-735.

25. Dunne DW, Vennervald BJ, Booth M, Joseph S, Fitzsimmons CM, et al. (2006) Applied and basic research on the epidemiology, morbidity, and immunology of schistosomiasis in fishing communities on Lake Albert, Uganda. Transactions of the Royal Society of Tropical Medicine and Hygiene 100: 216-223.

26. Kabatereine NB, Kemijumbi J, Ouma JH, Sturrock RF, Butterworth AE, et al. (2003) Efficacy and side effects of praziquantel treatment in a highly endemic Schistosoma mansoni focus at Lake Albert, Uganda. Transactions of the Royal Society of Tropical Medicine and Hygiene 97: 599-603. 
27. Zhang Y, Koukounari A, Kabatereine N, Fleming F, Kazibwe F, et al. (2007) Parasitological impact of 2-year preventive chemotherapy on schistosomiasis and soil-transmitted helminthiasis in Uganda. BMC Medicine 5: 27.

28. Kazibwe F, Makanga B, Rubaire-Akiiki C, Ouma J, Kariuki C, et al. (2009) Transmission studies of intestinal schistosomiasis in Lake Albert, Uganda and experimental compatibility of local Biomphalaria spp. Parasitology International 59: 49-53.

29. Kazibwe F, Makanga B, Rubaire-Akiiki C, Ouma J, Kariuki C, et al. (2006) Ecology of Biomphalaria (Gastropoda: Planorbidae) in Lake Albert, Western Uganda: snail distributions, infection with schistosomes and temporal associations with environmental dynamics. Hydrobiologia 568: 433-444.

30. WHO (2006) Preventive Chemotherapy in Human Helminthiasis. Coordinated use of Anthelminthic Drugs in Human Interventions: a Manual for Health Professionals and Programme Managers. Geneva: World Health Organization.

31. van Dam GJ, Wichers JH, Ferreira TMF, Ghati D, van Amerongen A, et al. (2004) Diagnosis of schistosomiasis by reagent strip test for detection of circulating cathodic antigen. Journal of Clinical Microbiology 42: 5458-5461.

32. Standley CJ, Lwambo NJS, Lange CN, Kariuki HC, Adriko M, et al. (2010) Performance of circulating cathodic antigen (CCA) urine-dipsticks for rapid detection of intestinal schistosomiasis in schoolchildren from shoreline communities of Lake Victoria. Parasites \& Vectors 3: 7

33. Katz N, Chavez A, Pellegrino J (1972) A simple device for quantitative stool thicksmear technique in schistosomiasis mansoni. Revista do Instituto de Medicina Tropical de São Paulo 14: 397-400.

34. Wilson RA, van Dam GJ, Kariuki TM, Farah IO, Deelder AM, et al. (2006) Detection limits for estimates of infection intensity in schistosomiasis mansoni established by a study in non-human primates. International Journal for Parasitology 36: 1241-1244.

35. Cringoli G, Rinaldi L, Utzinger J (2010) FLOTAC: new multivalent techniques for qualitative and quantitative copromicroscope diagnosis of parasites in animals and humans. Nature Protocols 5: 503-515.

36. Ihaka R, Gentleman R (1996) R: A language for data analysis and graphics. Journal of Computational and Graphical Statistics 5: 299-314.

37. Armitage P, Berry G (1994) Statistical Methods in Medical Research. Oxford: Blackwell Scientific Publications.

38. Kirkwood BR, Sterne JA (2003) Essential Medical Statistics (2nd edition). Oxford: Blackwell Science.
39. Bosompem KM, Bentum IA, Otchere J, Anyan WK, Brown CA, et al. (2004) Infant schistosomiasis in Ghana: a survey in an irrigation community. Tropical Medicine and International Health 9: 917-922.

40. Beck L, Van-Lume DSM, Souza JR, Domingues ALC, Favre T, et al. (2008) Discriminating acute from chronic human schistosomiasis mansoni. Acta Tropica 108: 229-233.

41. Andrade ZA (2009) Schistosomiasis and liver fibrosis. Parasite Immunology 31: 656-663.

42. Chiavaroli R, Grima P (2008) Detection of early liver fibrosis in patients with intestinal schistosomiasis: Sonographic and histologic findings in Schistosoma mansoni infection. Infection 36: 585-589.

43. Wilson S, Jones FM, Mwatha JK, Kimani G, Booth M, et al. (2009) Hepatosplenomegaly associated with chronic malaria exposure: evidence for a pro-inflammatory mechanism exacerbated by schistosomiasis. Parasite Immunology 31: 64-71.

44. Balen J, Stothard JR, Kabatereine NB, Tukahebwa EM, Kazibwe F, et al. (2006) Morbidity due to Schistosoma mansoni: an epidemiological assessment of distended abdomen syndrome in Ugandan school children with observations before and 1year after anthelminthic chemotherapy. Transactions of the Royal Society of Tropical Medicine and Hygiene 100: 1039-1048.

45. Medley G, Anderson RM (1985) Density-dependent fecundity in Schistosoma mansoni infections in man. Transactions of the Royal Society of Tropical Medicine and Hygiene 79: 532-534.

46. Monrad J, Christensen NO, Nansen P, Johansen MV, Lindberg R (1995) Acquired resistance against Schistosoma bovis after single or repeated low-level primary infections in goats. Research in Veterinary Science 58: 42-45.

47. Stothard JR, Kabatereine NB, Tukahebwa EM, Kazibwe F, Rollinson D, et al. (2006) Use of circulating cathodic antigen (CCA) dipsticks for detection of intestinal and urinary schistosomiasis. Acta Tropica 97: 219-228.

48. van Lieshout L, Polderman AM, Deelder AM (2000) Immunodiagnosis of schistosomiasis by determination of the circulating antigens CAA and CCA, in particular in individuals with recent or light infections. Acta Tropica 77: 69-80.

49. Bradley DJ, May RM (1978) Consequences of helminth aggregation for the dynamics of schistosomiasis. Transactions of the Royal Society of Tropical Medicine and Hygiene 72: 262-273. 